\title{
Editor's Comment
}

\author{
Kiyoshige Ohseto
}

\subsection{Thorax and Back Comment}

The nerve block for the posterior aspect of the thoracic spine can be safely performed using an interventional radiologycomputed tomography (IVR-CT) device, which combines computed tomography (CT) guidance and radiography and other devices. However, as the sympathetic nerves located in the upper thoracic spinal region are situated over the rib sections that are anatomically distant from the thoracic vertebrae, achieving the effect of sympathetic nerve block in the upper thorax is difficult. Therefore, endoscopic thoracic sympathectomy, in which sympathetic nerves are resected under direct thoracoscopic view, achieves immediate and long-term effects. 